\title{
SEASHELLS: THE PLAINNESS AND BEAUTY OF THEIR MATHEMATICAL DESCRIPTION
}

\author{
JORGE PICADO
}

\begin{abstract}
One might at first tend to think that the growth of plants and animals, because of their elaborate forms, are ruled by highly complex laws. However, this is surprisingly not always true: many aspects of the growth of plants and animals may be described by remarkably simple mathematical laws. An obvious example of this are the seashells and snails, as we show here: with a very simple model it is possible to describe and generate any of the many types of seashells of the classes of Gastropods, Bivalves, Cephalopods and Scaphopods that one may find classified in a good seashell bookguide.
\end{abstract}

«Beauty of style and harmony and grace and good rhythm depends on simplicity.»

- Plato

«There is much beauty in nature's clues, and we can all recognize it without any mathematical training. There is beauty, too, in the mathematical stories that start from the clues and deduce the underlying rules and regularities, but it is a different kind of beauty, applying to ideas rather than things. Mathematics is to nature as Sherlock Holmes is to evidence.» - I. Stewart 8

\section{How SEASHELLS GROW}

The idea that mathematics is deeply implied in the natural forms goes back to the Ancient Greeks. Many aspects of the growth of plants and animals may be described by remarkably simple mathematical laws, in spite of their elaborate forms (cf., for instance, the classical book of D'Arcy Thompson [9] and the recent book of Stephen Wolfram [10]).

An obvious example of this are the seashells and snails [6]. Why do so many shells form spirals? As far as the animal that lives in a shell grows he needs the shell to grow with him in order to keep accommodating him. The fact that the animal which lives at the open edge of the shell places new shell material always in that edge, and faster on one side than the other, makes the shell to grow in a spiral. The rates at which shell material is secreted at different points of the open edge are presumably determined by the anatomy of the animal. And, surprisingly, even fairly small changes in such rates can have quite tremendous effects on the overall shape of the shell, which is in the origin of the existence of a great diversity of shells.

Date: March 15, 2009. 
A two-dimensional version of this fact may be observed in the growth of horns. Like nails and hair, a horn grows by the addition of material as its base. In order to get a perfectly straight structure, the rate at which material is added must be exactly the same on each side of the base:

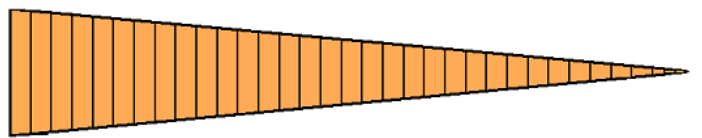

On the other hand, if there is some difference (indicated in percentage, in the figures below), one of the edges of the horn will be longer than the other and, inevitably, the horn will coil to the side where less material is added, following a spiral curve:

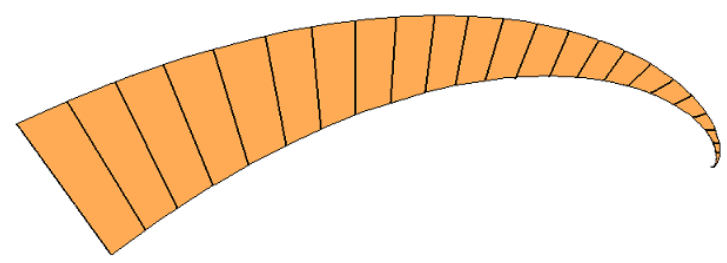

$10 \%$

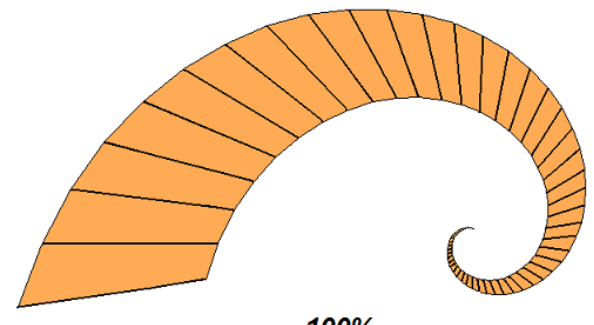

$100 \%$

It is essentially a three-dimensional version of this phenomena that yields the spiral structures of the shells of mollusks. Besides that, the mollusk does not enlarge its shell in a uniform way: it only adds material in one of the edges of the shell (the open or "growth" ending) and makes it in such a way that the new shell is always an exact model, to scale, of the smaller shell. This growth process yields an elegant spiral structure (very visible when the shell is sliced). The widths of the straight lines that link the shell center (the spiral origin) to the points of the shell increase, but the amplitudes of the angles defined by those lines and the corresponding tangents to the shell are constant, that is, shells follow an equiangular spiral:
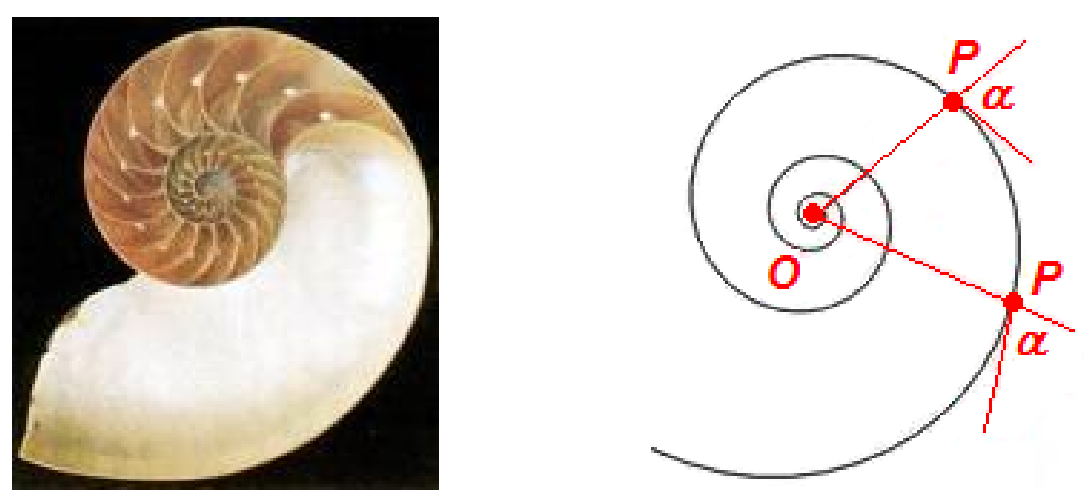

This fact was identified in the 17th century by Christopher Wren. A clear mathematical model of shell growth modes based on equiangular spirals was given by Henry Moseley 
in 1838, and the model used here is a direct extension of his (M. B. Cortie [1]). Careful studies from the mid-1800s to mid-1900s validated Moseley's basic model for a wide variety of shells.

As indicated in the figure above, given a point $O$ (the origin or the pole of the spiral), an equiangular spiral is a curve such that the angle $\alpha$ (the equiangular angle) between the tangent in each point $P$ of the curve and the radial line $O P$ is constant. Jacob Bernoulli (1654-1705) called it the Spira mirabilis (the marvellous spiral). It was first described mathematically in 1638 by René Descartes (who believed that "only mathematics is certain, so everything must be based on mathematics"). Its parametric equation ${ }^{1}$ is given, in polar coordinates $r$ and $\theta$, by

$$
r(\theta)=A e^{\theta \cot \alpha}, \quad \theta \geq 0,
$$

where $A$ denotes the radius associated to $\theta=0$. It gives the distance of a curve point to origin $O$ in terms of $\theta$. In cartesian coordinates, the points $(x(\theta), y(\theta))$ of the spiral are given by

$$
\left\{\begin{array}{l}
x(\theta)=r(\theta) \cos \theta \\
y(\theta)=r(\theta) \sin \theta
\end{array}\right.
$$

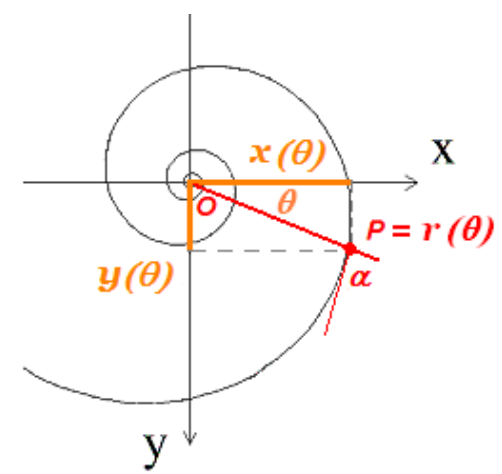

Note that when $\alpha=90^{\circ}$ the equiangular spiral degenerates to a circle. Of course the animal would not be very satisfied with a circular shell, because he could not keep growing inside the shell. If $\alpha$ is not a right angle, then a true spiral forms, which corresponds to an enlargement of the shell.

This growth process keeps the shape of the shell and is called gnomonic. In geometry, the gnomon (a word of Greek origin, due to Aristotle, meaning "indicator") of a given picture is a second picture that, added or subtracted to the former, generates a third picture similar to the original one. This growth pattern is so common that it is referred by many as a "law of nature". The majority of animal horns and nails, corals and snails, among other examples, also follow, basically, equiangular spirals.

The next figure shows three of the cases that may occur. The first example is typical of a cone, the second one of the nautilus shell and the third one of the clam of a bivalve. On the right, an horizontal section - in the case of the nautilus and the bivalve - and a vertical section - in the case of the cone - show the corresponding growth spiral. In each case the new shell material is progressively added in the aperture of the shell.

\footnotetext{
${ }^{1}$ Equivalently, the equation may be given by $\log (r(\theta) / A)=\theta \cot \alpha$. By that reason, the equiangular spiral is also known as the logarithmic spiral.
} 
(1) Cone:
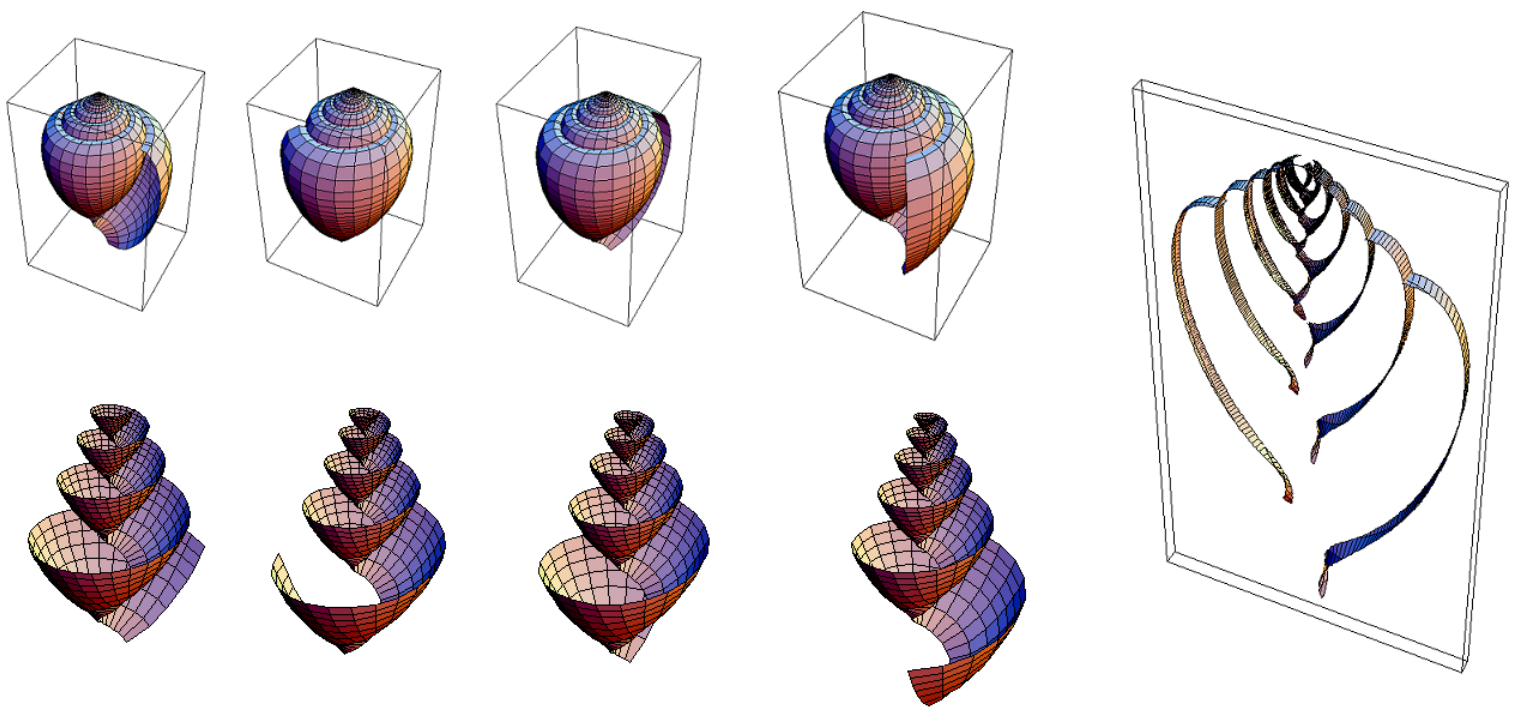

(2) Nautilus:
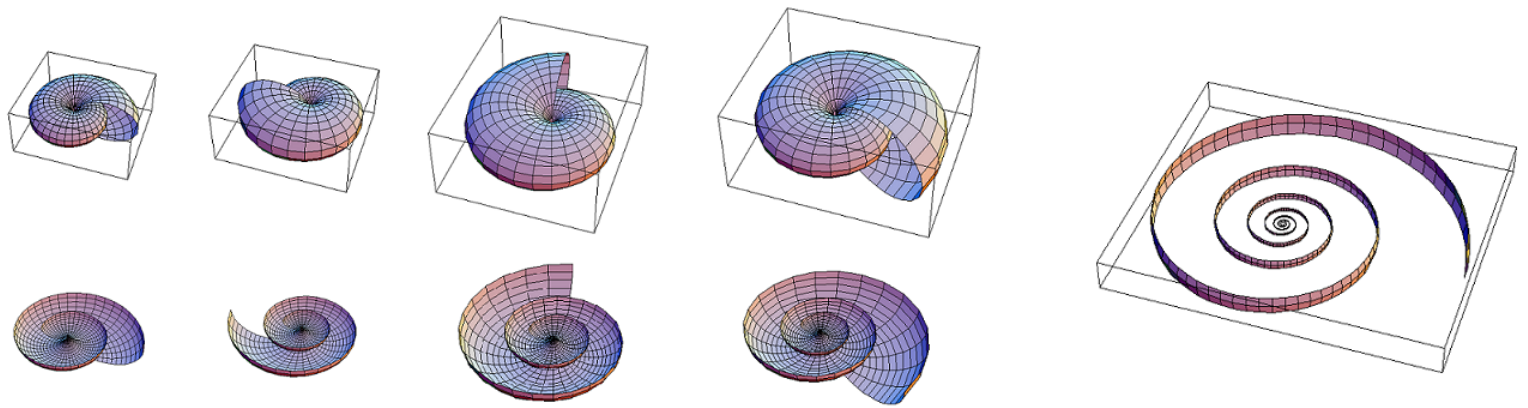

(3) Bivalve:
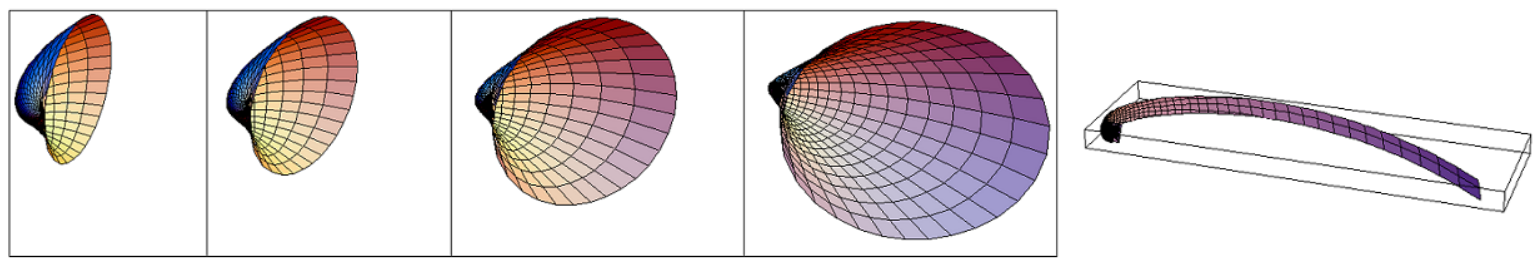

As we shall see, seashells, with their auto-similar shape, may be represented by a threedimensional surface, generated by a simple equation, with some free parameters. Amazingly, in spite of the simplicity of that equation, it is possible to generate a great variety of seashell types. Which ones? All of them! (with a very few exceptions: some live and fossil species of Vermicularia and fossil ammonites of the class of Didymoceras.) This shows how many of the 
forms that appear in nature are simple consequences of the application of three-dimensional geometry to the basic rules of growth.

«Mathematics is very much like poetry... what makes a good poem - a great poem - is that there is a large amount of thought expressed in very few words. In this sense formulas like $e^{\pi i}+1=0$ or $\int_{-\infty}^{\infty} e^{-x^{2}} d x=\sqrt{\pi}$ are poems.»

- LiPMAN Bers

\section{THE MODEL}

The surface of a shell is a three-dimensional surface that may be regarded as the result of the deplacement of a curve $\mathcal{C}$ (the generating curve, usually an ellipse) along an helico-spiral $\mathcal{H}$ (the structural curve) $([1],[4) ;$ the width of $\mathcal{C}$ increases as far as it moves along $\mathcal{H}$ :

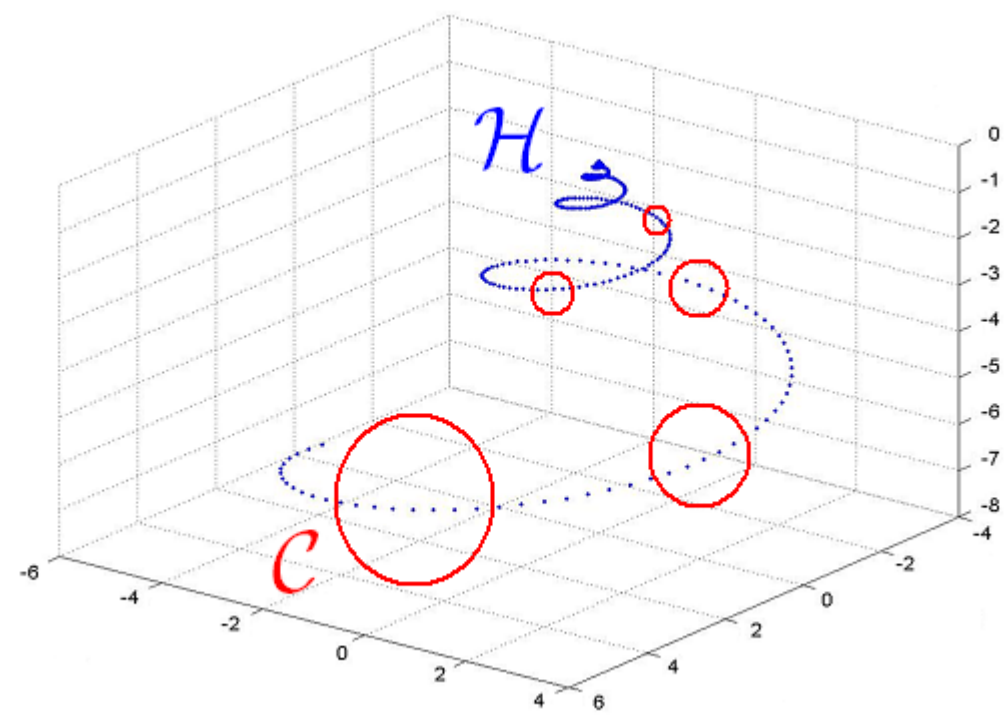

Why is $\mathcal{H}$ an helico-spiral? Basically because the mollusk does not enlarge its shell in a uniform manner: it secretes shell material faster on one side than the other of the open edge of the shell.

The shape of $\mathcal{C}$ describes the outline of the shell sections and of the shell aperture while $\mathcal{H}$ determines the global shape of the shell. $\mathcal{C}$ is not always an ellipse. This is the case of the beautiful Japanese Wonder shell, which is generated by a triangular curve: 


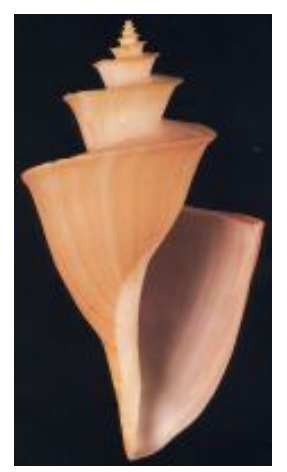

The effect of $\mathcal{C}$ in the final shape of the shell is illustrated in Section 4 (see also [7] for more details).

The first step in the construction of the model is to fix a three-dimensional cartesian system of coordinates $X Y Z$ and to consider the parametric equation of the helico-spiral, written in polar coordinates $r$ and $\theta$ :

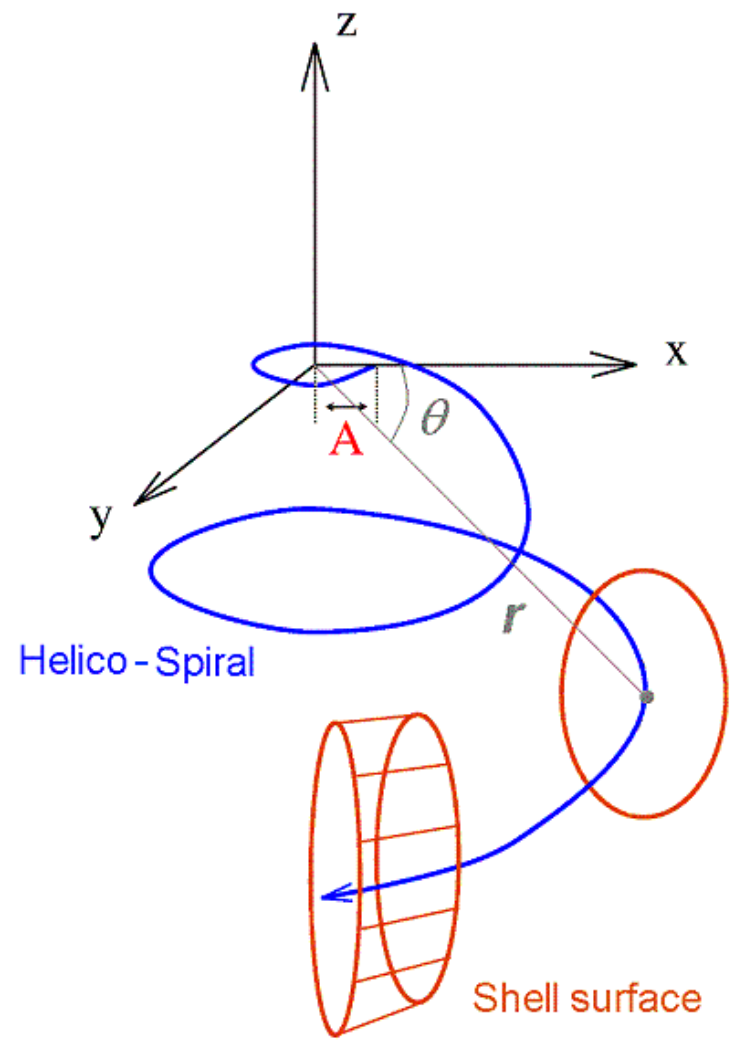

Let $\alpha$ denote the equiangular angle of spiral $\mathcal{H}, \beta$ the enlarging angle of spiral $\mathcal{H}$ (that is, the angle between $Z$-axis and the line from aperture local origin to $X Y Z$ origin) and let $A$ be the size of the spiral aperture (that is, the distance from main origin of aperture at $\theta=0)$ : 
Top view:

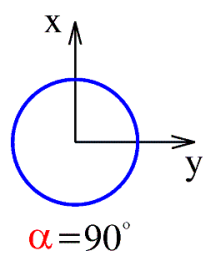

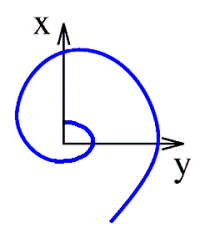

$\alpha=60^{\circ}$

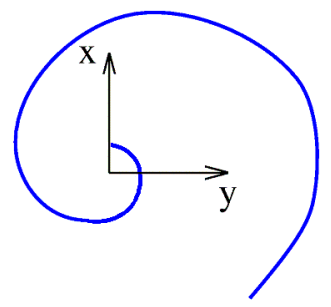

$\alpha=30^{\circ}$
Side view:

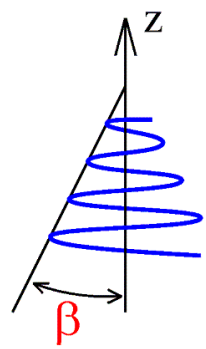

Seen from above the helico-spiral $\mathcal{H}$ looks like a logarithmic spiral. So, using (1.1), we assume that, for each $\theta \geq 0$, the distance $r(\theta)$ of the corresponding point $(x(\theta), y(\theta), z(\theta))$ of $\mathcal{H}$ to the origin is given by $r(\theta)=A e^{\theta \cot \alpha}$. Then

$$
z(\theta)=-r(\theta) \cos \beta
$$

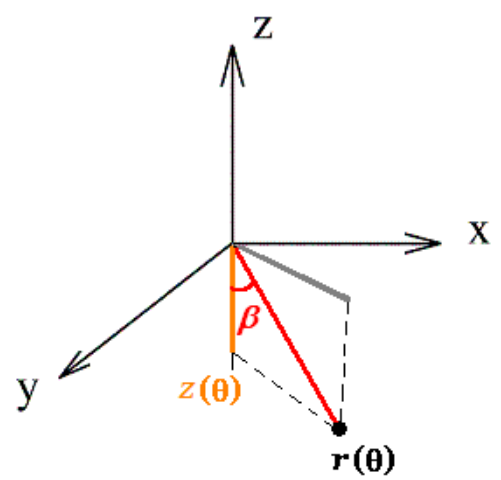

and

$$
\left\{\begin{array}{l}
x(\theta)=r(\theta) \sin \beta \cos \theta \\
y(\theta)=r(\theta) \sin \beta \sin \theta
\end{array}\right.
$$

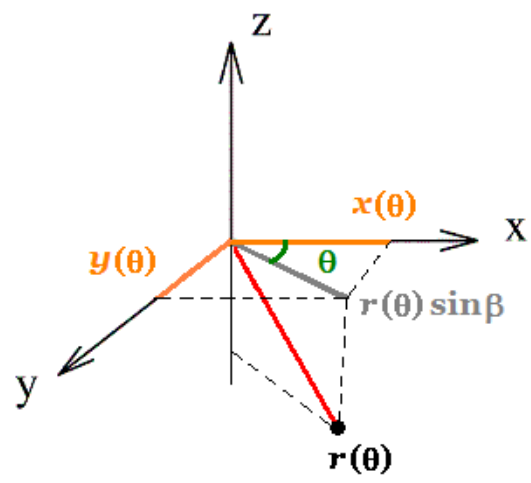

Therefore, the points $\mathcal{H}(\theta)=(x(\theta), y(\theta), z(\theta))$ of the helico-spiral satisfy equations

$$
\mathcal{H}(\theta)=\left\{\begin{array}{l}
x(\theta)=A \sin \beta \cos \theta e^{\theta \cot \alpha} \\
y(\theta)=A \sin \beta \sin \theta e^{\theta \cot \alpha} \\
z(\theta)=-A \cos \beta e^{\theta \cot \alpha} .
\end{array}\right.
$$


The generating curve $\mathcal{C}$ that determines the surface of the shell is, in most cases, an ellipse with parameters $a$ (semimajor axis) and $b$ (semiminor axis),

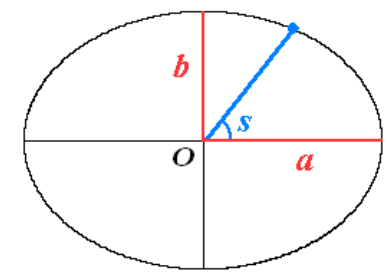

that is, a curve with parametric equation

$$
r_{e}(s)=\frac{a b}{\left[(a \sin s)^{2}+(b \cos s)^{2}\right]^{\frac{1}{2}}}=\left[\left(\frac{\cos s}{a}\right)^{2}+\left(\frac{\sin s}{b}\right)^{2}\right]^{-\frac{1}{2}}, 0 \leq s \leq 2 \pi .
$$

The width of $\mathcal{C}$ increases as far as it moves along $\mathcal{H}$. We assume that its increasing rate $r_{i}(\theta)$ is the same as the one of $\mathcal{H}$, that is, $r_{i}(\theta)=e^{\theta \cot \alpha}$. Then, the equation of each $\mathcal{C}$ in polar coordinates (centered at the corresponding $\mathcal{H}(\theta)$ ) is given by

$$
R_{e}(\theta, s)=r_{e}(s) r_{i}(\theta)=r_{e}(s) e^{\theta \cot \alpha}, \theta \geq 0,0 \leq s \leq 2 \pi .
$$

Equivalently, in terms of cartesian coordinates:

$$
\mathcal{C}(\theta, s)=\left\{\begin{array}{l}
x^{\mathcal{C}}(\theta, s)=\cos s \cos \theta r_{e}(s) e^{\theta \cot \alpha} \\
y^{\mathcal{C}}(\theta, s)=\cos s \sin \theta r_{e}(s) e^{\theta \cot \alpha} \\
z^{\mathcal{C}}(\theta, s)=\sin s r_{e}(s) e^{\theta \cot \alpha}
\end{array}\right.
$$

Finally, to obtain the equation of the shell $\mathfrak{S}$, it suffices to put the equations 2.3 of $\mathcal{C}$ in the corresponding points of $\mathcal{H}$ (ruled by equations (2.1)):

$$
\mathfrak{S}(\theta, s)=\mathcal{H}(\theta)+\mathcal{C}(\theta, s)=\left\{\begin{array}{l}
x^{\mathfrak{S}}(\theta, s)=\left(A \sin \beta \cos \theta+\cos s \cos \theta r_{e}(s)\right) e^{\theta \cot \alpha} \\
y^{\mathfrak{S}}(\theta, s)=\left(A \sin \beta \sin \theta+\cos s \sin \theta r_{e}(s)\right) e^{\theta \cot \alpha} \\
z^{\mathfrak{S}}(\theta, s)=\left(-A \cos \beta+\sin s r_{e}(s)\right) e^{\theta \cot \alpha}
\end{array}\right.
$$

We can now easily extend this model to the more general situation where the generating ellipse $\mathcal{C}$ rotates in space. For this we specify three angles $\phi, \Omega, \mu$ that establish the orientation of $\mathcal{C}$ in space. They measure the rotation of the curve around, respectively, a vector orthogonal to the plane of the ellipse, the $O Z$ axis and its horizontal axis, as the following picture illustrates: 


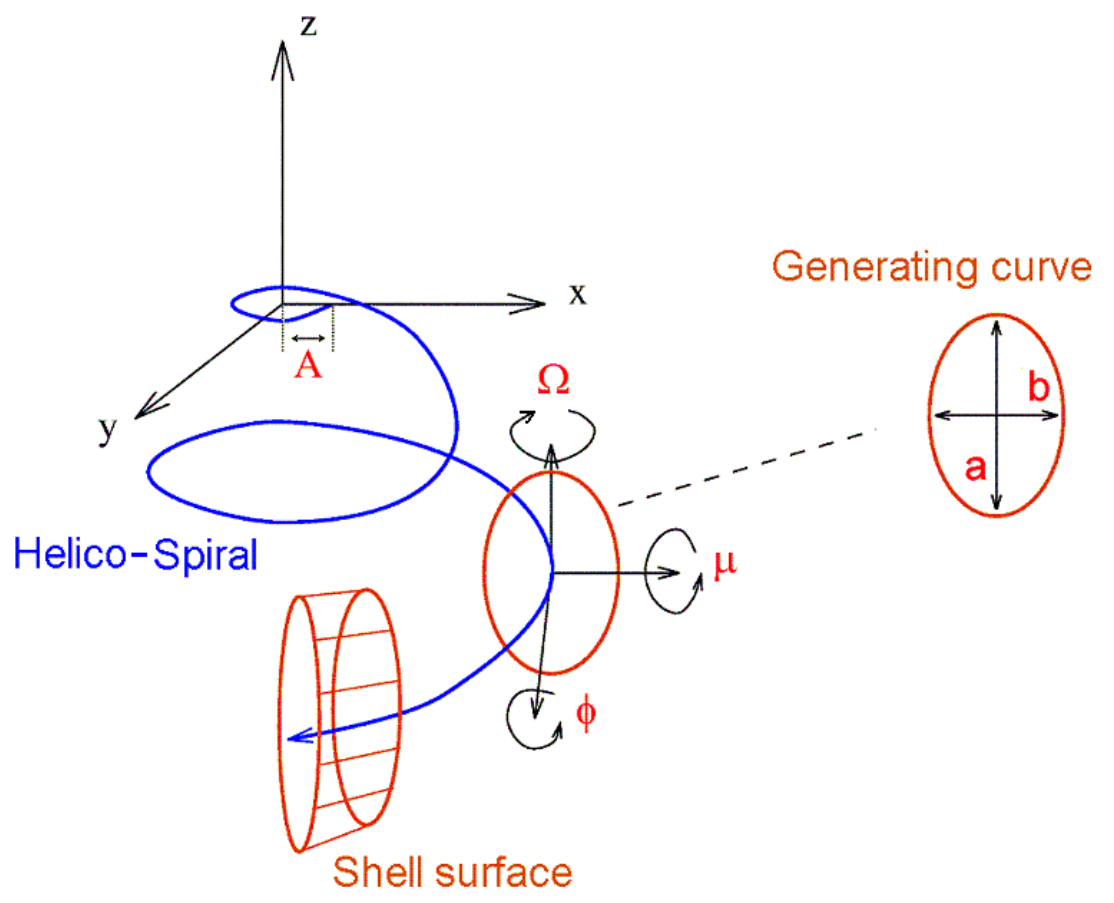

- Case 1 (rotation $\phi$ ): it suffices to replace $s$ by $s+\phi$ in each sin and cos function in (2.3).

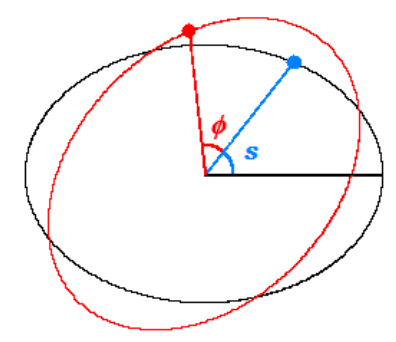

- Case 2 (rotation $\Omega$ ): it suffices to replace $\theta$ by $\theta+\Omega$ in each sin and cos function in (2.3).

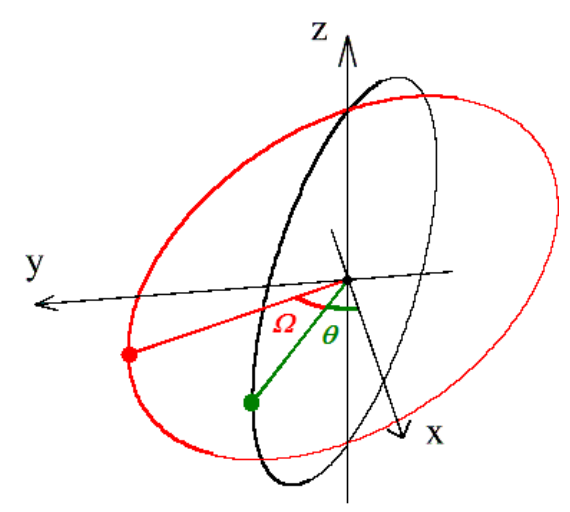

Thus, equations (2.3) are replaced by 


$$
\mathcal{C}_{2}(\theta, s)=\left\{\begin{array}{l}
x_{2}^{\mathcal{C}}(\theta, s)=\cos (s+\phi) \cos (\theta+\Omega) r_{e}(s) e^{\theta \cot \alpha} \\
y_{2}^{\mathcal{C}}(\theta, s)=\cos (s+\phi) \sin (\theta+\Omega) r_{e}(s) e^{\theta \cot \alpha} \\
z_{2}^{\mathcal{C}}(\theta, s)=\sin (s+\phi) r_{e}(s) e^{\theta \cot \alpha}
\end{array}\right.
$$

that is, the equations of $(2.4)$ of the shell are now given by

$$
\mathfrak{S}_{2}(\theta, s)=\left\{\begin{array}{l}
x_{2}^{\mathfrak{S}}(\theta, s)=\left(A \sin \beta \cos \theta+\cos (s+\phi) \cos (\theta+\Omega) r_{e}(s)\right) e^{\theta \cot \alpha} \\
y_{2}^{\mathfrak{S}}(\theta, s)=\left(A \sin \beta \sin \theta+\cos (s+\phi) \sin (\theta+\Omega) r_{e}(s)\right) e^{\theta \cot \alpha} \\
z_{2}^{\mathfrak{S}}(\theta, s)=\left(-A \cos \beta+\sin (s+\phi) r_{e}(s)\right) e^{\theta \cot \alpha} .
\end{array}\right.
$$

- Case 3 (rotation $\mu$ ): Observing the ellipse $\mathcal{C}_{2}$ by profile and the result of its rotation of angle $\mu$, let $\mathcal{C}_{3}(\theta, s)=\left(x_{3}^{\mathcal{C}}(\theta, s), y_{3}^{\mathcal{C}}(\theta, s), z_{3}^{\mathcal{C}}(\theta, s)\right)$ be the point on the new ellipse $\mathcal{C}_{3}$ corresponding to $\mathcal{C}_{2}(\theta, s)$ :

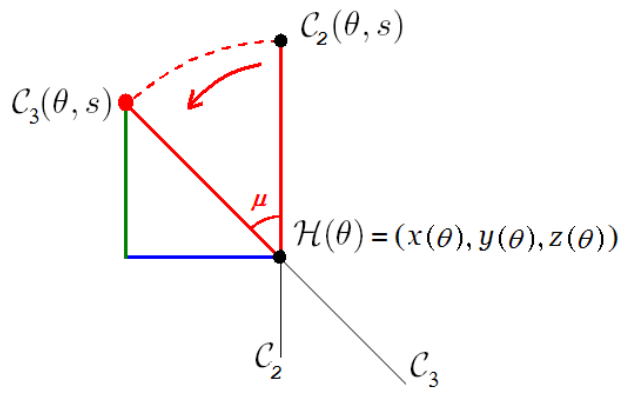

Then

$$
z_{3}^{\mathcal{C}}(\theta, s)=z(\theta)+z_{2}^{\mathcal{C}}(\theta, s) \cos \mu=\left(-A \cos \beta+\cos \mu \sin (s+\phi) r_{e}(s)\right) e^{\theta \cot \alpha} .
$$

Further, looking from above we have

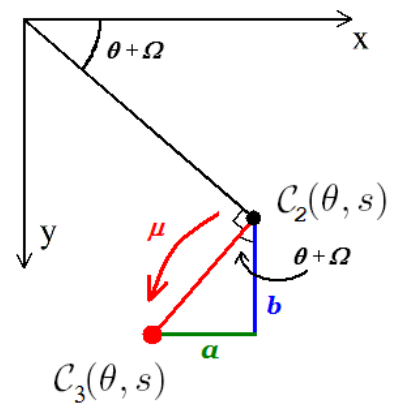

from which we conclude that

$$
\begin{aligned}
& x_{3}^{\mathcal{C}}(\theta, s)=x_{2}^{\mathfrak{S}}(\theta, s)-a=x_{2}^{\mathfrak{S}}(\theta, s)-\left[z_{2}^{\mathcal{C}}(\theta, s) \sin \mu\right] \sin (\theta+\Omega) \\
& y_{3}^{\mathcal{C}}(\theta, s)=y_{2}^{\mathfrak{S}}(\theta, s)+b=y_{2}^{\mathfrak{S}}(\theta, s)+\left[z_{2}^{\mathcal{C}}(\theta, s) \sin \mu\right] \cos (\theta+\Omega) .
\end{aligned}
$$


Finally, taking also into account the direction $D$ of coiling ( 1 if dextral and -1 if sinistral), we get the parametric equations that describe the surface of a shell generated by an ellipse $\mathcal{C}$, with equation $r_{e}(s)$, moving along an helico-spiral (D. Fowler, H. Meinhardt and P. Prusinkiewicz [4], M. B. Cortie [1] $)^{2}$

$$
\begin{aligned}
& x_{3}^{\mathfrak{S}}(\theta, s)= \\
& \quad D\left[A \sin \beta \cos \theta+\cos (s+\phi) \cos (\theta+\Omega) r_{e}(s)-\sin \mu \sin (s+\phi) \sin (\theta+\Omega) r_{e}(s)\right] e^{\theta \cot \alpha} \\
& y_{3}^{\mathfrak{S}}(\theta, s)= \\
& \quad\left[A \sin \beta \sin \theta+\cos (s+\phi) \sin (\theta+\Omega) r_{e}(s)+\sin \mu \sin (s+\phi) \cos (\theta+\Omega) r_{e}(s)\right] e^{\theta \cot \alpha} \\
& z_{3}^{\mathfrak{S}}(\theta, s)=\left[-A \cos \beta+\cos \mu \sin (s+\phi) r_{e}(s)\right] e^{\theta \cot \alpha} .
\end{aligned}
$$

\section{The COMPlete MODEL: ALLOWING NODUlES AND SPIKES}

In case we want to add nodules, bumps and spikes to the shell, it suffices to insert some additional parameters: $N$ (the number of nodules along a complete revolution of $\theta$ ), $W_{1}$ (the length of each nodule along the generating curve), $W_{2}$ (the length of each nodule along the helico-spiral), $L$ (the height of each nodule) and $P$ (the angle that indicates the position of the nodule in the generating curve):

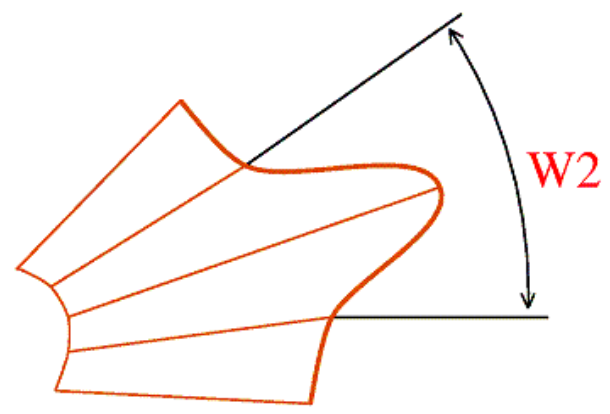

Top view

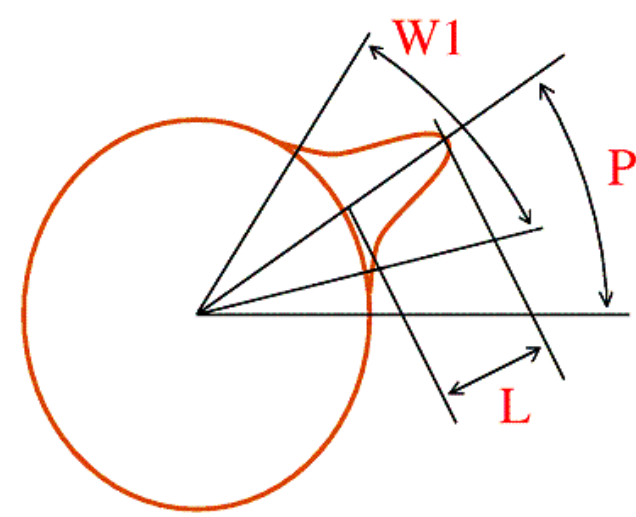

Side view

From [2] (cf. [5]), if a surface sculpture term is superimposed in the ellipse $\mathcal{C}$, it suffices to replace the equation $r_{e}(s)$ of the ellipse by $r_{e}(s)+r_{n}(s, \theta),(0 \leq s \leq 2 \pi, \theta \geq 0)$, where

\footnotetext{
${ }^{2}$ Alternatively, $\mathcal{C}$ may be any other planar curve such as, for example, a cardioid with equation $r_{e}(s)=$ $a(1-\cos s)$.
} 
$r_{n}(s, \theta)=0$ in case $W_{1}=0$ or $W_{2}=0$ or $N=0$, and

$$
r_{n}(s, \theta)=L e^{-\left[\left(\frac{2(s-P)}{W_{1}}\right)^{2}+\left(\frac{2 l(\theta)}{W_{2}}\right)^{2}\right]} \quad \text { with } l(\theta)=\frac{2 \pi}{N}\left[\frac{N \theta}{2 \pi}-\operatorname{int}\left(\frac{N \theta}{2 \pi}\right)\right],
$$

otherwist 3 .

In summary, the equations of the shell depend on 14 parameters (the latter four are only of relevance in case $L$ is nonzero):

$$
D, A, \alpha, \beta, \mu, \Omega, \phi, a, b, L, P, W_{1}, W_{2}, N \text {. }
$$

«Mathematics, rightly viewed, possesses not only truth, but supreme beauty - a beauty cold and austere, like that of sculpture, without appeal to any part of our weaker nature... sublimely pure, and capable of a stern perfection such as only the greatest art can show.»

- Bertrand Russell

\section{EXAMPLES}

The model above, incorporating (3.1), can be easily written in Mathematica in the following way

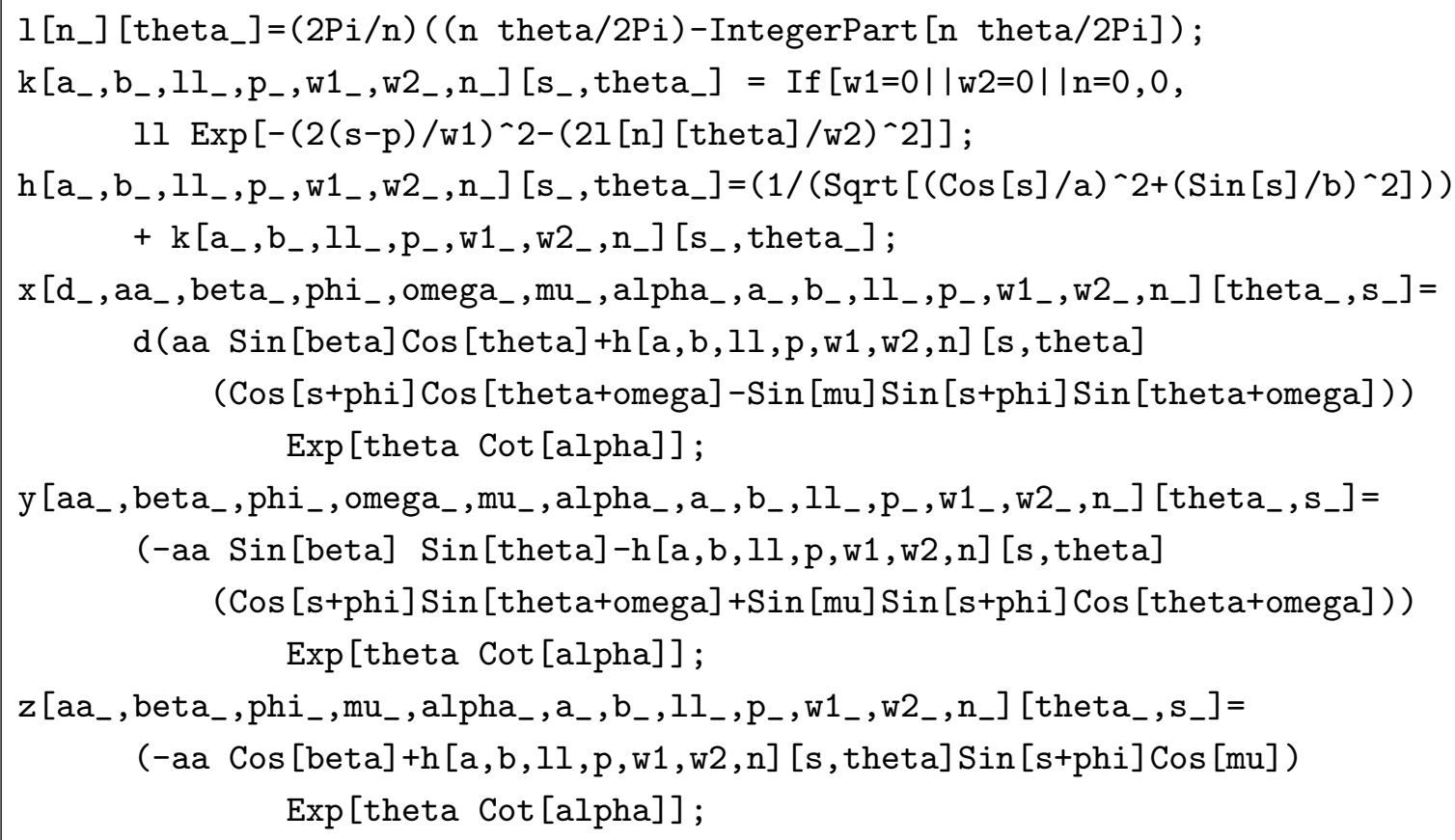

\footnotetext{
${ }^{3}$ Note that this model contains the previous case of shells with no nodules: it suffices to make $L=0$.

${ }^{4}$ Since the $X Y Z$ referential we use in our model has opposite orientation to the one used by Mathematica, we change the sign in coordinate $y$.
} 
By choosing appropriate values for the various parameters and ranging $\theta$ from $\theta$ min to $\theta \max$ and $s$ from smin to $\operatorname{smax}$

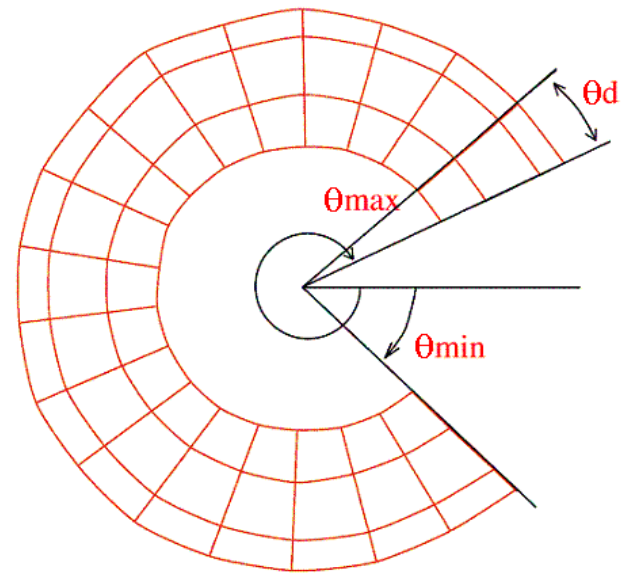

Top view

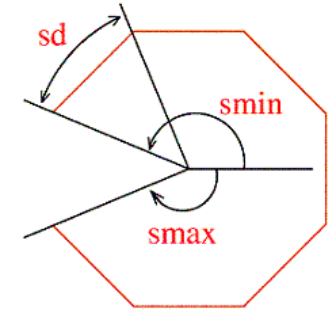

Side view

we can now plot the surface of any type of shell.

For example, the Boat Ear Moon (cf. [3], p. 78), from the class of Gastropods, can be generated by parameters $D=1, A=25, \alpha=83^{\circ}, \beta=42^{\circ}, \mu=10^{\circ}, \Omega=30^{\circ}, \phi=70^{\circ}$, $a=12, b=20, L=0, P=0, W_{1}=1, W_{2}=1$ and $N=1$ :
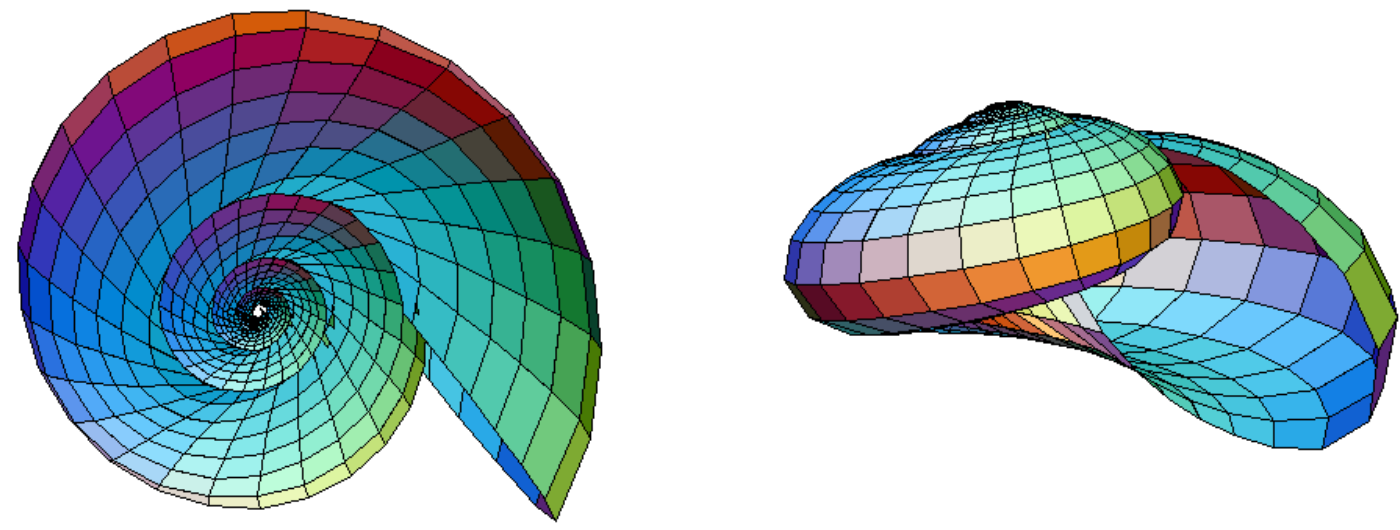

With $[\{d=1, a a=25$, beta=42Degree, phi=70Degree, omega=30Degree, mu=10Degree, alpha=83Degree, $a=12, b=20,11=0, p=0, w 1=0, w 2=0, n=0\}$,

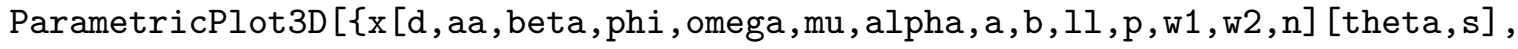
y [aa, beta, phi , omega , mu, alpha , a , b , ll, p, w1 , w2 , n] [theta , s] , z [aa, beta , phi , mu , alpha, a , b , 11, p, w1, w2, n] [theta , s]\}, \{theta, -4Pi, 4Pi\}, \{s, -270 Degree, 90 Degree , Boxed $\rightarrow$ False, Axes $\rightarrow$ False, PlotPoints $\rightarrow\{100,20\}$, PlotRange $\rightarrow$ All, ViewPoint $\rightarrow\{-1,-3,0.5\}]]\}$ 
We conclude with a few more examples of shells that may be found in [3] and that can be modelled this way (in [7] you may manipulate the figures, with more detail). The most surprising example is the one of bivalves, with their umbones (protuberances in the top) wonderfully plotted!

The Precious Wentletrap

$\left(D=1, A=90, \alpha=86^{\circ}, \beta=10^{\circ}, \mu=5^{\circ}, \Omega=1^{\circ}, \phi=-45^{\circ}, a=20, b=20, L=14, P=40\right.$, $\left.W_{1}=180, W_{2}=0.4, N=180\right)$ :
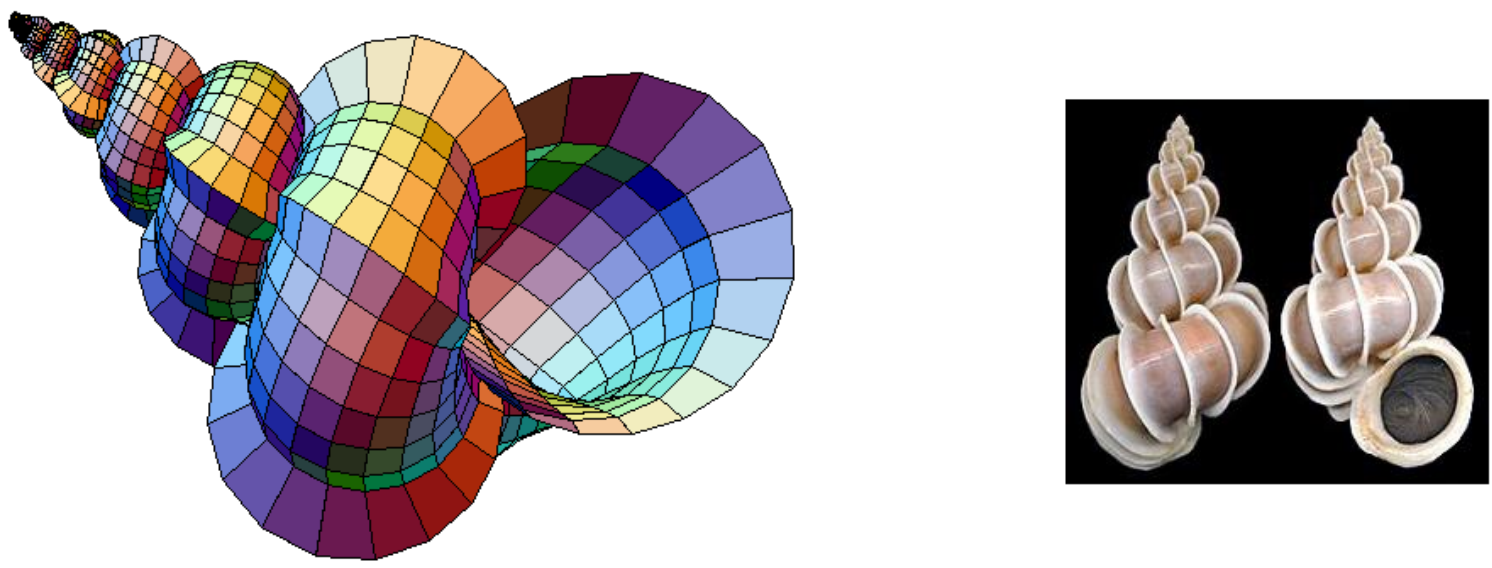

Turritella, ancilla and whelk:
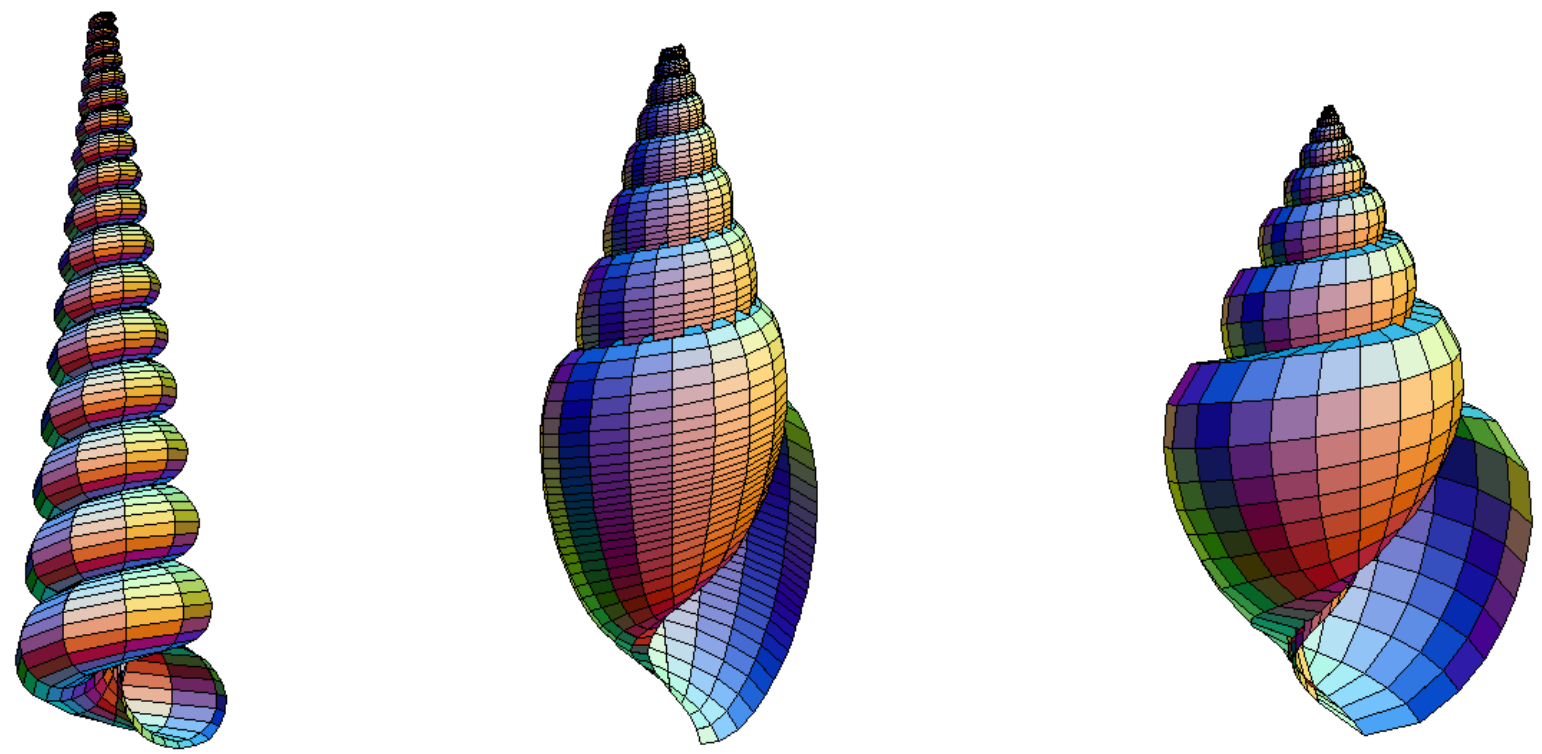
Two top shells (the Tiger Maurea and the Commercial Trochus):
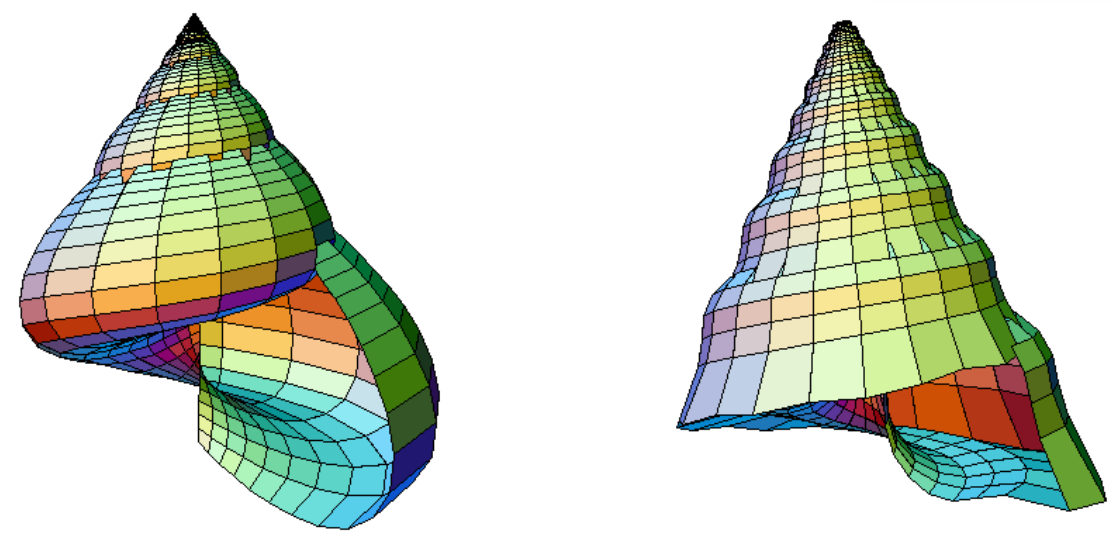

Tun shell, olive and horse conch:
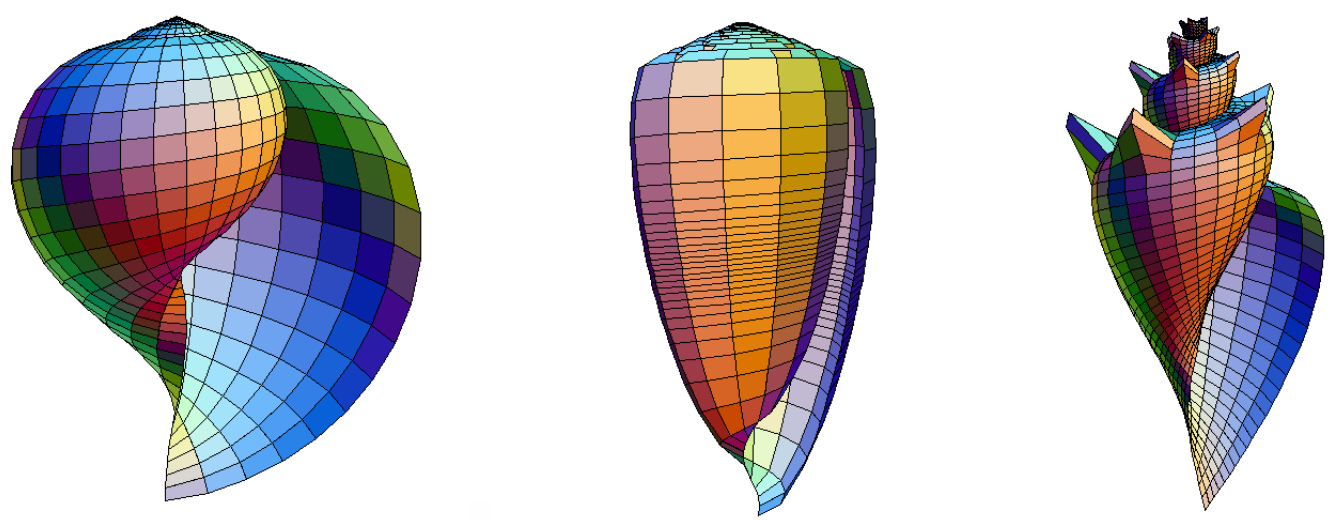

Two limpets (the Common European Limpet and the Blue-rayed Limpet):
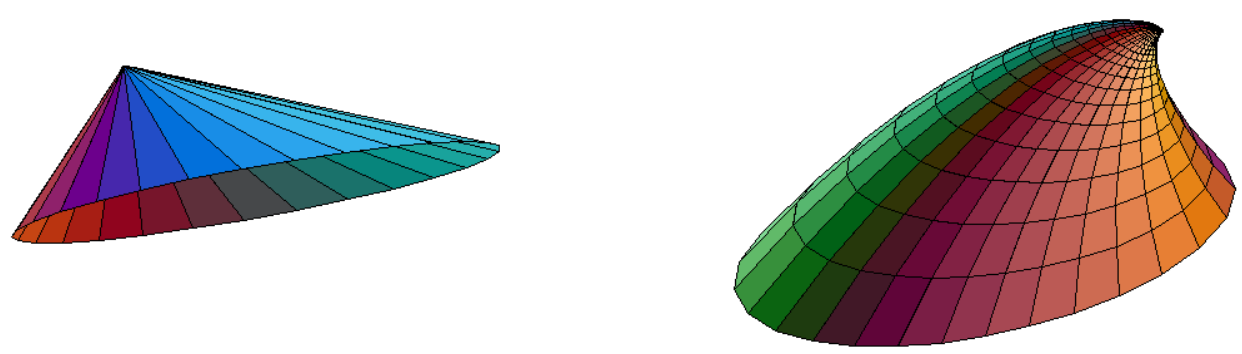
Two bivalves (the European Glycymeris and the Lyonsia):
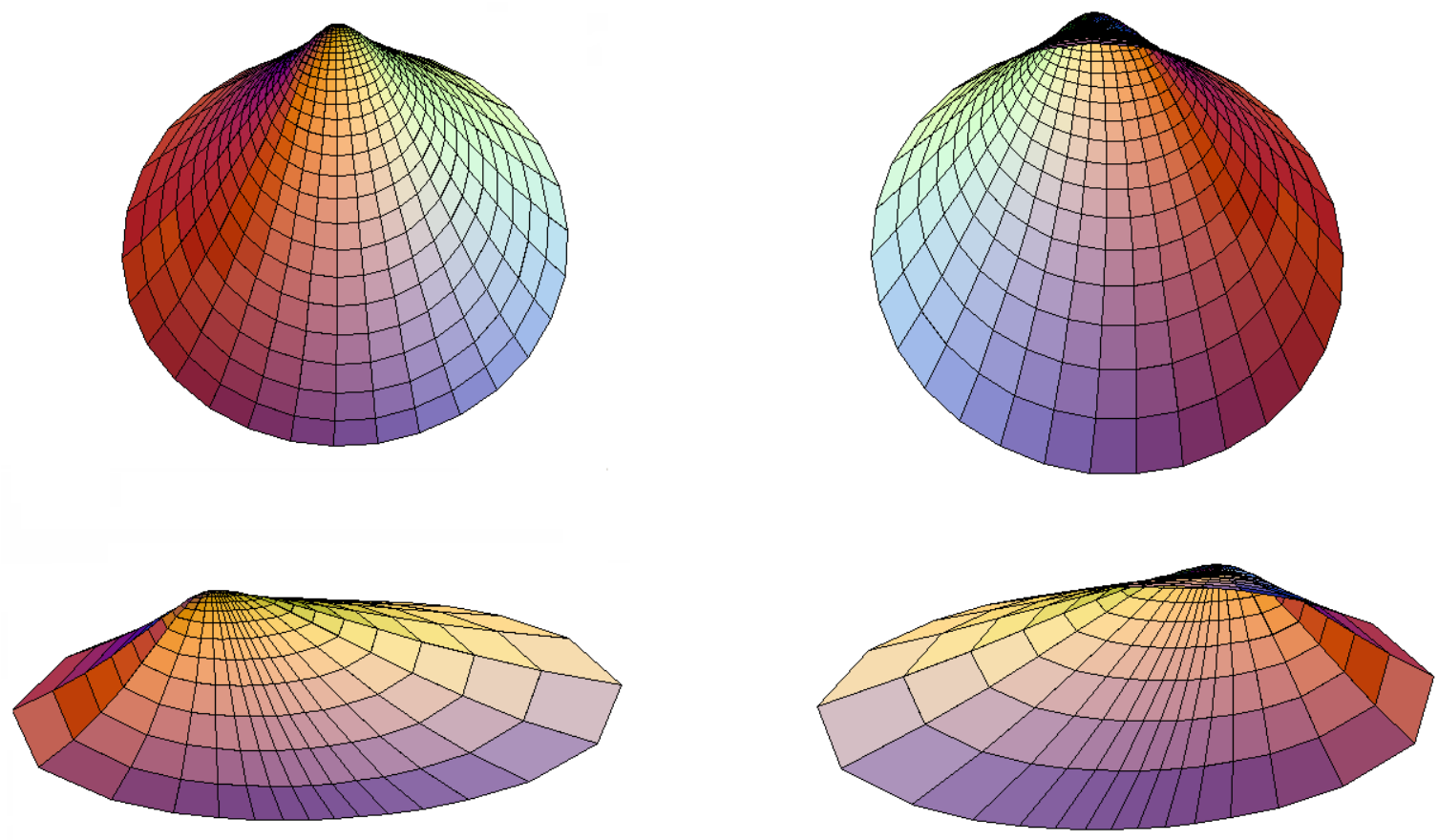

Argonauta (Cephalopod):
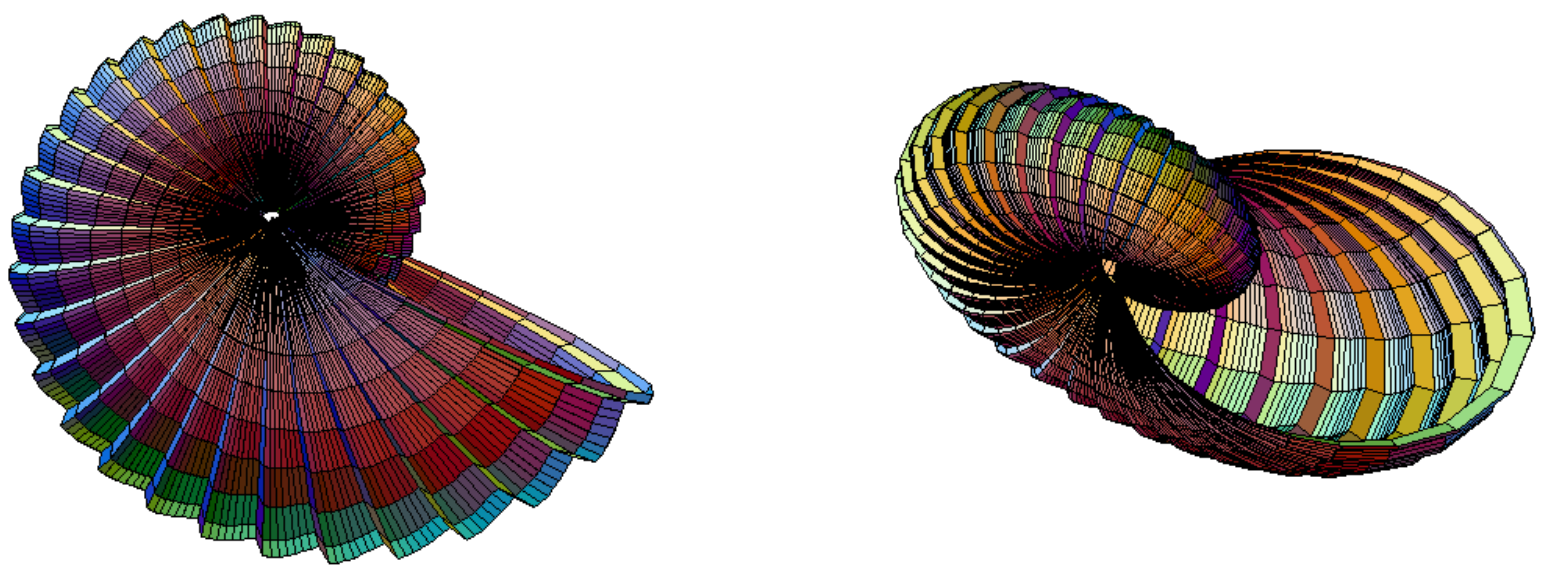
Worm shell, elephant tusk (Scaphopod) and spirula:

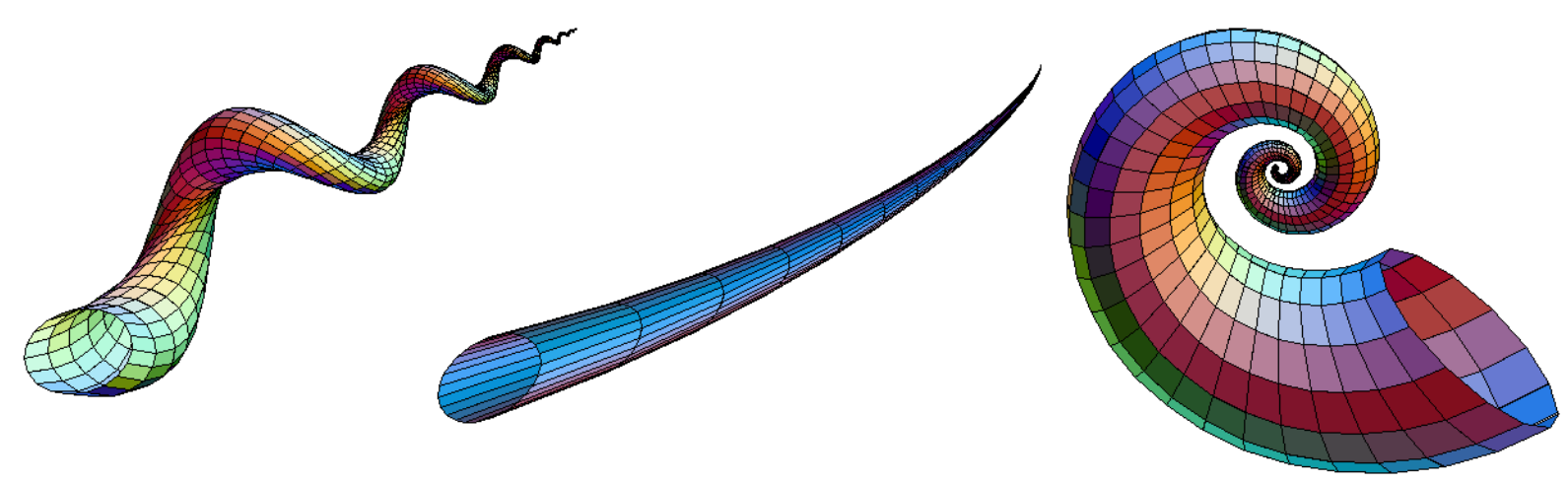

Planorbis and ammonite (fossil):
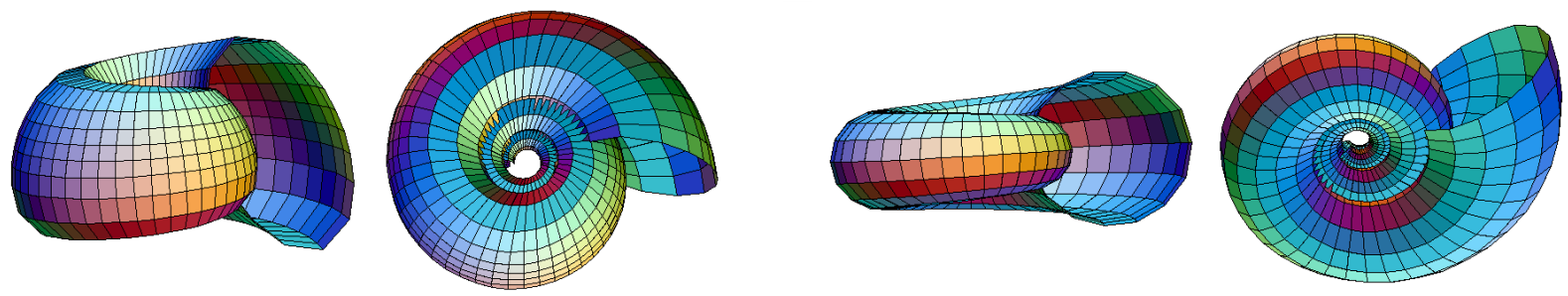

«The scientist does not study nature because it is useful; he studies it because he delights in it, and he delights in it because it is beautiful. If nature were not beautiful, it would not be worth knowing, and if nature were not worth knowing, life would not be worth living.»

- HenRi POINCARÉ

\section{REFERENCES}

[1] M. B. Cortie, Digital seashells, Comput. \& Graphics 17 (1993) 79-84.

[2] M. B. Cortie, Modelling the surface bumps and spikes of molluscan shells, in: Proceedings of the First International Conchology Conference (Ed. C.R. Illert), Hadronic Press, Palm Harbor, 1995, pp. 46-65.

[3] S. P. Dance, Shells: The Photographic Recognition Guide to Seashells of the World, DK Publ., 2002.

[4] D. Fowler, H. Meinhardt and P. Prusinkiewicz, Modeling seashells, Computer Graphics 26 (1992) 379387.

[5] J. Lega, Natural patterns. Lecture 8: modeling sea shells, November 1999, math.arizona.edu/ lega/ 195/Fal199/lectnotes/lect8/index.htm

[6] H. Meinhardt, The Algorithmic Beauty of Sea Shells, Springer Verlag, 1998.

[7] J. Picado, Seashells: the plainness and beauty of their mathematical description, www.mat.uc.pt/ $\sim$ picado/conchas. 
[8] I. Stewart, Nature's Numbers: Discovering Order and Pattern in the Universe, Science Masters, Orion Publishing Group, 1998.

[9] D'A. Thompson, On Growth and Form, Cambridge University Press, 1961.

[10] S. Wolfram, A New Kind of Science, Wolfram Research, Inc., 2004.

Centre for Mathematics of the University of Coimbra

3001-454 CoImbra, PORTUGAL

E-mail address: picado@mat.uc.pt

$U R L$ : www .mat.uc.pt/ picado 